\title{
Malondialdeído e troponina I cardíaca em equinos da raça Puro Sangue Árabe submetidos ao exercício e à suplementação com vitamina $\mathrm{E}$
}

\author{
Malondialdehyde and cardiac troponin I in Arabian horses subjected to exercise and \\ vitamin E supplementation
}

\author{
Letícia Andreza Yonezawa' ${ }^{\mathrm{I}}$ Luciana Pereira Machado ${ }^{\mathrm{II}}$ Veridiana Fernandes da Silveira ${ }^{\text {III }}$ \\ Marcos Jun Watanabe ${ }^{\mathrm{IV}}$ Mere Erika Saito $^{\mathrm{V}}$ Sandra Satiko Kitamura $^{\mathrm{VI}}$ Aguemi Kohayagawa $^{\mathrm{VII}}$
}

\section{RESUMO}

Para avaliar o efeito do exercício físico, do treinamento e da suplementação com vitamina E sobre o malondialdeído (MDA) e a troponina I cardíaca (cTnI) séricos, foram utilizados 16 equinos da raça Puro Sangue Árabe, sem treinamento, divididos em dois grupos de oito: controle e suplementado com vitamina E. Ambos os grupos foram submetidos a provas de exercício progressivo em esteira de alta velocidade inclinada a $+7 \%$, antes (P1) e após (P2) o período de treinamento de 20 dias. Foram determinadas as concentrações séricas de MDA, vitamina E e cTnI e realizou-se o eletrocardiograma de repouso antes e após as provas $P 1 e$ $P 2$. Os resultados sugerem a ocorrência do estresse oxidativo ocasionado pelo exercício, determinado por meio do aumento de MDA sérico. Em alguns animais, houve um discreto aumento de cTnI associado à detecção de complexos ventriculares prematuros, mas que não representou lesão cardíaca expressiva. Não houve efeito do treinamento sobre o estresse oxidativo. Portanto, é possível concluir que o exercício físico progressivo promoveu a lipoperoxidação nessas condições, que não foi prevenida pela suplementação com vitamina $E$ nem pelo treinamento, mas não foi suficiente para causar uma lesão cardíaca significativa.

Palavras-chave: estresse oxidativo, metabolismo cardíaco, cavalo, medicina esportiva.

\section{ABSTRACT}

To evaluate the effect of exercise, training and vitamin E supplementation on serum malondialdehyde (MDA) and cardiac troponin I (cTnI), sixteen adult untrained Arabian horses were divided into 2 groups of 8 animals each, control and supplemented with vitamin E. Both groups had incremental exercise tests performed on a high-speed treadmill at a $+7 \%$ slope, before (P1) and after (P2) a training period of 20 days. Serum concentrations of MDA, vitamin E and CTnI, and resting electrocardiogram were performed before and after $P 1$ and $P 2$. Results suggested that oxidative stress was induced by exercise as measured by serum MDA increase. In some animals, there was a subtle increase in cTnI concentration associated with the detection of ventricular premature complexes, which didn't represent a significant cardiac injury. There was no training effect on oxidative stress. It was concluded that, under the conditions above, the incremental exercise promoted lipoperoxidation, which was not prevented by vitamin $E$ supplementation or training, however, it was not enough to cause a significant cardiac damage.

Key words: oxidative stress, cardiac metabolism, horse, sports medicine.

\section{INTRODUÇÃO}

O exercício físico é capaz de promover estresse oxidativo induzido pelo desequilíbrio entre a produção de Espécies Reativas de Oxigênio (ERO) e a capacidade antioxidante das células e dos fluidos extracelulares, ocasionando lipoperoxidação das

IPrograma de Pós-graduação Medicina Veterinária, Faculdade de Medicina Veterinária e Zootecnia (FMVZ), Universidade Estadual Paulista (Unesp), Campus de Botucatu, Distrito de Rubião Junior, s/n, 18618-000, Botucatu, SP, Brasil. E-mail: leticiay@gmail.com. Autor para correspondência.

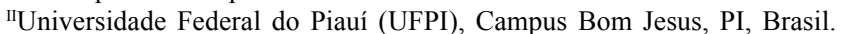

IIIUniversidade Federal do Recôncavo da Bahia (UFRB), Cruz das Almas, BA, Brasil.

IVDepartamento de Cirurgia e Anestesiologia Veterinária, FMVZ, Unesp, Botucatu, SP, Brasil.

${ }^{\mathrm{V}}$ Departamento de Medicina Veterinária, Universidade do Estado de Santa Catarina (UDESC), Lages, SC, Brasil.

VIUniversidade Anhembi-Morumbi, São Paulo, SP, Brasil.

${ }^{\mathrm{VII} D e p a r t a m e n t o ~ d e ~ C l i ́ n i c a ~ V e t e r i n a ́ r i a, ~ F M V Z, ~ U n e s p, ~ B o t u c a t u, ~ S P, ~ B r a s i l . ~}$ 
membranas celulares e lesão tecidual (WILLIAMS \& CARLUCCI et al., 2006). Considerando o papel potencialmente deletério da produção excessiva de ERO e a evidência de que o exercício induz o estresse oxidativo em equinos, a suplementação com antioxidantes objetiva restabelecer ou manter o equilíbrio oxidante/antioxidante e parece ser uma perspectiva promissora para o bem-estar animal e desempenho atlético. Como a deficiência de antioxidantes, o estresse oxidativo induzido e a consequente intolerância ao exercício físico já foram claramente estabelecidos, o oposto, ou seja, a suplementação de antioxidantes para melhoria do desempenho atlético, ainda precisa ser comprovado (DE MOFFARTS et al., 2005).

McMENIMAN \& HINTZ (1992), avaliando pôneis submetidos a exercício em esteira e suplementados com vitamina E, observaram uma correlação negativa entre a vitamina E plasmática e os índices de lipoperoxidação, demonstrando a prevenção do estresse oxidativo ocasionado pelo exercício físico por esse composto. Entretanto, WILLIAMS et al. (2004) não verificaram benefícios da suplementação com vitamina E sobre o estresse oxidativo ocasionado pelo exercício de enduro.

Apesar das evidências diretas da ocorrência de estresse oxidativo e de lipoperoxidação, a vitamina E assume um papel determinante na proteção do coração contra situações deletérias decorrentes do exercício agudo (VENDITTI et al., 1999). O músculo cardíaco apresenta um alto metabolismo aeróbico, exibindo uma elevada demanda de oxigênio em condições de repouso, que aumenta em grandes proporções durante um exercício físico exaustivo. Essa alta taxa de metabolismo oxidativo é geralmente associada com a produção acrescida de ERO (ATALAY \& SEN, 1999). Contudo, no coração, a capacidade antioxidante parece ser limitada, tornando-o susceptível à lesão tecidual por estresse oxidativo após um período de exercício agudo (ASCENSÃO et al., 2003).

A troponina cardíaca foi descrita como um marcador altamente específico e sensível para dano miocárdico em muitas espécies de mamíferos, e sua estrutura é muito similar entre elas (YONEZAWA et al., 2010). Apesar do mecanismo responsável pelo aumento da troponina associado ao exercício ainda ser desconhecido, um estudo em ratos revela evidência histológica de lesão miocárdica localizada e inflamação no ventrículo esquerdo 24 a $48 \mathrm{~h}$ após serem submetidos ao exercício intenso de natação, e a concentração de troponina $\mathrm{T}$ cardíaca foi proporcionalmente superior nos animais que executaram esforço mais acentuado. Mesmo que os autores não souberam explicar os mecanismos envolvidos nessa lesão miocárdica(CHEN et al., 2000), sabe-se que podem causar instabilidade elétrica e aumento do potencial arritmogênico do músculo cardíaco (LEROUX et al., 1995). NEUMAYR et al. (2002) sugerem que, em atletas humanos após exercício físico, há lesão na membrana celular do cardiomiócito, aumentando a concentração de troponina I cardíaca (cTnI) na circulação. Entretanto, essa lesão é transitória e reversível, provavelmente ocasionada por uma isquemia durante o exercício e pelo aumento da permeabilidade decorrente do estresse oxidativo induzido pela sobrecarga de ERO (WHYTE et al., 2000; NEUMAYR et al., 2002), permitindo que somente a fração citosólica de 2,8 a $8,3 \%$ da cTnI total presente no tecido cardíaco seja liberada. Entretanto, a significância desse compartimento citosólico é desconhecida para a espécie equina (HOLBROOK et al., 2006).

O presente estudo objetivou avaliar o efeito do exercício físico, do treinamento e da suplementação com vitamina E em equinos da raça Puro Sangue Árabe sobre o estresse oxidativo, avaliado por meio da mensuração do malondialdeído e vitamina E séricos, e sobre a lesão cardíaca, determinada pela troponina I cardíaca sérica.

\section{MATERIAL E MÉTODOS}

Foram utilizados 16 equinos adultos, da raça Puro Sangue Árabe, sendo três machos castrados e 13 fêmeas, com idade variando de 4,5 a 12 anos, peso médio de $362,3 \pm 25,6 \mathrm{~kg}$, sem treinamento físico prévio, clinicamente sadios e selecionados mediante exames físico e complementares. Esses foram alojados em piquetes sob as mesmas condições de manejo alimentar e sanitário. A alimentação consistiu de feno de capim coast-cross, ração comercial, suplemento mineral e água ad libitum. Os equinos foram distribuídos em dois grupos de oito animais: Controle (GC) e Suplementado com Vitamina E (dl-alfa-tocoferol) (GE). A suplementação com vitamina $\mathrm{E}$ via oral, por meio de cápsula gelatinosa $\left(\right.$ E-Tabs ${ }^{\circledR}$, Sigma Pharma, Hortolândia, SP, Brasil) misturada a melaço de cana, no grupo GE, teve início 15 dias antes da primeira prova de exercício (P1), na dose de 1.000 UI animal ${ }^{-1}$, diariamente, sem interrupção até o final do experimento.

Foram realizadas duas provas de exercício progressivo, denominadas de P1 (antes do treinamento) e P2 (após o treinamento), as quais foram realizadas em esteira de alta velocidade (Mustang 2200 AG - Kagra, Suíça) inclinada a $+7 \%$. O exercício iniciou-se a $5 \mathrm{~min}$ no passo a $1,8 \mathrm{~m} \mathrm{~s}^{-1}$, seguido de $3 \mathrm{~min}$ no trote a $4,0 \mathrm{~m} \mathrm{~s}^{-1}$, $2 \mathrm{~min}$ no cânter a $6,0 \mathrm{~m} \mathrm{~s}^{-1} \mathrm{e}$ em seguida $1 \mathrm{~min}$ no galope 
a $8,0 \mathrm{~m} \mathrm{~s}^{-1}, 9,0 \mathrm{~m} \mathrm{~s}^{-1}, 10,0 \mathrm{~m} \mathrm{~s}^{-1}, 11,0 \mathrm{~m} \mathrm{~s}^{-1}$ ou até quando o animal pudesse manter-se em exercício, mesmo sendo estimulado, dependendo da capacidade individual (MACHADO et al., 2007). Os animais foram treinados, entre P1 e P2, uma vez ao dia, seis vezes por semana, até completarem 20 dias. O protocolo de treinamento adotado foi de $5 \mathrm{~min}$ a $1,8 \mathrm{~m} \mathrm{~s}^{-1}$ (passo), $3 \mathrm{~min}$ a $4,0 \mathrm{~m} \mathrm{~s}^{-1}$ (trote), $2 \mathrm{~min}$ a $6,2 \mathrm{~m} \mathrm{~s}^{-1}$ (cânter), $1 \mathrm{~min} \mathrm{a} 8,0 \mathrm{~m} \mathrm{~s}^{-1}$ e a $10,0 \mathrm{~m}$ $\mathrm{s}^{-1}$ (galope), seguidos de um período de desaquecimento a $3,0 \mathrm{~m} \mathrm{~s}^{-1}$ (trote) por $2 \mathrm{~min}$ e a $1,6 \mathrm{~m} \mathrm{~s}^{-1}$ (passo) por $2 \mathrm{~min}$ (MACHADO et al., 2007). Após o último dia de treinamento, os animais não foram submetidos à atividade física por $48 \mathrm{~h}$ antes de realizarem a segunda prova de exercício (P2).

Antes e após as provas, foi realizado o eletrocardiograma de repouso em oito animais, de acordo com a técnica descrita por FREGIN (1982), durante um minuto. Amostras de sangue foram coletadas para obtenção de soro antes do início do exercício (M0), imediatamente após, e 15min, 30min, $2 \mathrm{~h}, 6 \mathrm{~h}, 12 \mathrm{~h}$ e $24 \mathrm{~h}$ após o exercício em ambas as provas, e armazenadas a $-80^{\circ} \mathrm{C}$ até as análises. As amostras destinadas à determinação de vitamina $\mathrm{E}$ foram protegidas da luz. As concentrações séricas de MDAe vitamina $\mathrm{E}$ foram mensuradas por meio da cromatografia líquida de alta eficiência (HPLC), em fluoroscopia, de acordo com KARATAS et al. (2002) e ARNAUD et al. (1991), respectivamente. A concentração de cTnI foi determinada pela técnica "ELFA" (Ensaio fluorescente ligado à enzima) utilizando kit comercial (Vidas ${ }^{\circledR}$ Troponin I Ultra - bioMérieux Clinical Diagnostics, França).

De acordo com a distribuição dos dados, analisados pelo Teste de Kolgomorov e Smirnov, as variáveis foram avaliadas segundo métodos estatísticos paramétricos ou não-paramétricos. $\mathrm{Na}$ ocorrência do primeiro caso, os dados foram inicialmente avaliados por meio de teste $\mathrm{F}$ de análise de variância e, quando significativo, as médias foram confrontadas pelo teste de múltipla amplitude de Tukey. Todas as análises foram consideradas significativas quando $\mathrm{P}<0,05$.

\section{RESULTADOS E DISCUSSÃO}

Estudos relacionados à suplementação com antioxidantes e o estresse oxidativo no exercício físico são cada vez mais frequentes em virtude do maior interesse no bem-estar animal, desempenho atlético e conhecimento da fisiologia do exercício. Entretanto, os benefícios reais da utilização desses compostos e sua dose ótima recomendada ainda não foram totalmente elucidados, uma vez que há uma ampla variação dos resultados encontrados na literatura (AVELLINI et al., 1999; KIRSCHVINK et al., 2006; WILLIAMS \& CARLUCCI et al., 2006; WILLIAMS, 2008).

Avaliando o efeito do exercício progressivo, observou-se que o MDA apresenta um discreto aumento imediatamente após o exercício, exceto para o GC-P2, o qual foi acentuado em $15 \mathrm{~min}$, com pico máximo em 30min, exceto para GC-P1, que apresentou valor máximo em $15 \mathrm{~min}$. Porém, essa elevação foi significativa apenas em 15 e 30min do GE na $\mathrm{P} 2(\mathrm{P}<0,006)$. Os valores basais retornaram em $2 \mathrm{~h}$ após o exercício, com exceção do GE-P2, que retornou em 6h. A análise do efeito da suplementação entre GC e GE não revelou diferença estatística entre os grupos avaliados, porém observouse que os valores de MDA do GC foram superiores ao do GE em todos os momentos, exceto nos momentos em P2 de 15 e 30min após o exercício (Tabela 1).

AVELLINI et al. (1999) demonstraram que a suplementação com vitamina $\mathrm{E}$, associada a um período de treinamento de 70 dias, foi suficiente para aumentar os sistemas detoxificadores e reduzir as ERO, demonstrados pela diminuição dos níveis plasmáticos de MDA. Embora não tenha havido diferença significativa, no presente estudo, os animais do grupo suplementado com vitamina $\mathrm{E}$ apresentaram menores concentrações séricas de MDA, no repouso, imediatamente após e a partir de $2 \mathrm{~h}$ após o exercício, sugerindo que a suplementação foi benéfica. Contudo, a suplementação não preveniu o estresse oxidativo provocado pelo exercício, demonstrado pelo aumento de MDA em 15 e 30min, em ambos os grupos e provas, que foi significativo apenas no GE, na $\mathrm{P} 2(\mathrm{P}<0,006)$, mas foi capaz de impedir a propagação desse estresse, evidenciado pelo rápido retorno de MDA aos níveis basais em $2 \mathrm{~h}$. A maior produção de MDA no GE pode ser decorrente do efeito pró-oxidante da vitamina $\mathrm{E}$. Uma das funções da vitamina E é transformar uma ERO potencialmente nociva em compostos menos reativos. Nesse tipo de reação, a vitamina E é oxidada, podendo ser novamente reduzida para vitamina $E$ ativa e originar mais ERO. Esses oxidantes atravessam livremente as membranas lipídicas e, apesar de menos deletério que as demais ERO, em grande concentração, pode ultrapassar a capacidade de depuração, promovendo maior lesão (SETIADI et al., 2003; YEUM et al., 2004). WILLIAMS et al. (2004) e WILLIAMS \& CARLUCCI (2006) não encontraram diferenças significativas em índices de lipoperoxidação entre equinos suplementados com altas doses de vitamina $E$ e animais controle submetidos a exercícios de enduro e progressivo em esteira, respectivamente. 
Tabela 1 - Médias ( \pm desvios-padrão) da concentração sérica de malondialdeído (MDA) ( $\eta \mathrm{mol} / \mathrm{mL}$ ) de equinos da raça Puro Sangue Árabe nas provas P1 (antes do treinamento) e P2 (após o treinamento) de exercício progressivo em esteira, nos grupos controle (GC) e suplementado com vitamina $\mathrm{E}(\mathrm{GE})$, antes (M0), imediatamente após (PE) e 15min, 30min, 2h, 6h, 12h e 24h após o exercício.

\begin{tabular}{|c|c|c|c|c|c|c|c|c|c|c|}
\hline \multicolumn{11}{|c|}{ Momentos } \\
\hline \multirow{3}{*}{$\mathrm{GC}$} & & M0 & $\mathrm{PE}$ & $15 \mathrm{~min}$ & $30 \mathrm{~min}$ & $2 \mathrm{~h}$ & $6 \mathrm{~h}$ & $12 \mathrm{~h}$ & $24 \mathrm{~h}$ & Signif \\
\hline & $\mathrm{P} 1$ & $1,07 \pm 0,67$ & $1,15 \pm 0,94$ & $1,22 \pm 0,88$ & $1,18 \pm 0,88$ & $0,97 \pm 0,88$ & $0,90 \pm 0,85$ & $1,07 \pm 0,85$ & $0,90 \pm 0,79$ & $\mathrm{P}>0,99$ \\
\hline & $\mathrm{P} 2$ & $1,12 \pm 0,96$ & $1,11 \pm 0,96$ & $1,15 \pm 0,94$ & $1,25 \pm 0,99$ & $1,13 \pm 0,91$ & $0,98 \pm 0,89$ & $1,28 \pm 1,10$ & $0,89 \pm 0,70$ & $\mathrm{P}>0,99$ \\
\hline \multirow{2}{*}{ GE } & P1 & $0,66 \pm 0,51$ & $0,68 \pm 0,31$ & $0,73 \pm 0,38$ & $1,16 \pm 0,65$ & $0,62 \pm 0,30$ & $0,54 \pm 0,30$ & $0,59 \pm 0,30$ & $0,57 \pm 0,35$ & $\mathrm{P}>0,08$ \\
\hline & P2 & $0,54 \pm 0,31^{\mathrm{b}}$ & $0,65 \pm 0,34^{b}$ & $1,21 \pm 0,67^{\mathrm{a}}$ & $1,35 \pm 0,85^{\mathrm{a}}$ & $0,70 \pm 0,42^{\mathrm{b}}$ & $0,56 \pm 0,48^{b}$ & $0,48 \pm 0,34^{b}$ & $0,50 \pm 0,32^{b}$ & $\mathrm{P}<0,006$ \\
\hline \multicolumn{2}{|c|}{ Signif } & $\mathrm{P}>0,1$ & $\mathrm{P}>0,2$ & $\mathrm{P}>0,1$ & $\mathrm{P}>0,6$ & $\mathrm{P}>0,3$ & $\mathrm{P}>0,3$ & $\mathrm{P}>0,09$ & $\mathrm{P}>0,09$ & \\
\hline
\end{tabular}

Letras minúsculas distintas na mesma linha indicam diferença estatística entre momentos dentro do mesmo grupo. Signif: nível de significância.

Ao confrontar as provas de exercício progressivo, não houve diferença do MDA entre P1 e P2, com resultados semelhantes antes e após o treinamento em ambos os grupos, indicando que esse protocolo não foi capaz de amenizar a lipoperoxidação ocasionada pelo exercício. WILLIAMS et al. (2008) também não observaram alterações significativas em índice de lipoperoxidação com um protocolo de treinamento por oito semanas.

A concentração sérica da vitamina E sempre se manteve mais elevada no GE em relação ao GC, embora não tenha apresentado diferença estatística entre momentos, grupos e provas (Tabela 2). MCMENIMAN e HINTZ (1992) também verificaram concentrações significativamente maiores em pôneis suplementados e DE MOFFARTS et al. (2005) em equinos Puro Sangue Inglês com relação ao grupo controle. WILLIAMS \& CARLUCCI et al. (2006) observaram níveis superiores em cavalos de trote suplementados, mas estes apresentaram significância após $24 \mathrm{~h}$ do exercício em esteira, quando comparados aos animais controle. Esses autores também relatam que a vitamina E plasmática aumentou com o exercício e retornou ao nível basal após $1 \mathrm{~h}$ do término do teste em esteira. McMENIMAN \& HINTZ(1992) e DEATON et al. (2002) não encontraram diferença na concentração plasmática de vitamina $E$ em equinos suplementados $\mathrm{e}$ submetidos a exercício. No presente estudo, também não foram observadas grandes alterações nos níveis de vitamina $\mathrm{E}$ em consequência do exercício ou treinamento. A diminuição da vitamina $\mathrm{E}$ em $15 \mathrm{~min}$ coincide com o aumento da concentração de MDA, sugerindo o consumo desse antioxidante para evitar a propagação do estresse oxidativo.

Acredita-se que o estresse oxidativo ocasionado pelo exercício físico promove a lipoperoxidação e lesão de membrana dos cardiomiócitos em razão do aumento de ERO, favorecendo a liberação de cTnI do compartimento citosólico e seu aumento na circulação (WHYTE et al., 2000; NEUMAYR et al., 2002). No entanto, neste estudo, não foi possível correlacionar as concentrações séricas do indicador de lipoperoxidação, o MDA, com as de cTnI. Muitos trabalhos descrevem que a cTnI do miocárdio equino apresenta alta reação cruzada com os kits de imunoensaios humanos de cTnI, podendo ser utilizada como biomarcador de lesão de cardiomiócitos (YONEZAWA et al., 2010). A concentração sérica de cTnI, na maioria dos animais e momentos, permaneceu abaixo do limite de detecção

Tabela 2 - Médias ( \pm desvios-padrão) da concentração sérica de vitamina E ( $\mu \mathrm{mol} / \mathrm{mL})$ de equinos da raça Puro Sangue Árabe nas provas P1 (antes do treinamento) e P2 (após o treinamento) de exercício progressivo em esteira, nos grupos controle (GC) e suplementado com vitamina E (GE), antes (M0), imediatamente após (PE) e 15min, 30min, 2h, 6h, 12h e 24h após o exercício.

\begin{tabular}{|c|c|c|c|c|c|c|c|c|c|c|}
\hline \multicolumn{11}{|c|}{ Momentos } \\
\hline \multirow{3}{*}{$\mathrm{GC}$} & & M0 & $\mathrm{PE}$ & $15 \mathrm{~min}$ & $30 \mathrm{~min}$ & $2 \mathrm{~h}$ & $6 \mathrm{~h}$ & $12 \mathrm{~h}$ & $24 \mathrm{~h}$ & Signif \\
\hline & $\mathrm{P} 1$ & $2,95 \pm 0,98$ & $3,10 \pm 0,99$ & $2,78 \pm 0,79$ & $2,90 \pm 0,79$ & $2,74 \pm 0,76$ & $2,76 \pm 0,75$ & $3,07 \pm 0,95$ & $2,77 \pm 0,91$ & $\mathrm{P}>0,9$ \\
\hline & $\mathrm{P} 2$ & $2,93 \pm 0,75$ & $3,12 \pm 1,18$ & $2,96 \pm 0,96$ & $3,00 \pm 1,73$ & $2,82 \pm 0,77$ & $2,81 \pm 0,93$ & $3,04 \pm 1,21$ & $2,81 \pm 1,02$ & $\mathrm{P}>1,0$ \\
\hline \multirow{2}{*}{ GE } & P1 & $3,47 \pm 0,89$ & $3,68 \pm 0,99$ & $3,25 \pm 0,72$ & $3,17 \pm 0,77$ & $2,84 \pm 0,76$ & $2,84 \pm 0,56$ & $3,46 \pm 1,15$ & $3,14 \pm 0,73$ & $\mathrm{P}>0,4$ \\
\hline & $\mathrm{P} 2$ & $3,18 \pm 0,98$ & $3,67 \pm 1,24$ & $3,21 \pm 0,96$ & $3,01 \pm 0,84$ & $3,01 \pm 0,87$ & $3,12 \pm 0,91$ & $3,33 \pm 1,09$ & $3,25 \pm 1,09$ & $\mathrm{P}>0,9$ \\
\hline \multicolumn{2}{|c|}{ Signif } & $\mathrm{P}>0,3$ & $\mathrm{P}>0,2$ & $\mathrm{P}>0,2$ & $\mathrm{P}>0,5$ & $\mathrm{P}>0,6$ & $\mathrm{P}>0,5$ & $\mathrm{P}>0,5$ & $\mathrm{P}>0,4$ & \\
\hline
\end{tabular}

Signif: nível de significância.

Ciência Rural, v.40, n.6, jun, 2010. 
do kit de $<0,01 \mu \mathrm{g} \mathrm{L}^{-1}$. Entretanto, três animais do GE em P2 apresentaram níveis de cTnI superiores a esse valor de limite de detecção, provavelmente em razão do efeito pró-oxidante da vitamina $\mathrm{E}$, conforme descrito anteriormente. Observou-se aumento de cTnI no momento 6h após o exercício (animais 11, 15 e 16, respectivamente: 0,$03 ; 0,18$ e $\left.0,05 \mu \mathrm{g} \mathrm{L}^{-1}\right)$, retornando aos níveis basais $\left(<0,01 \mu \mathrm{g} \mathrm{L}^{-1}\right)$ nos animais $11 \mathrm{e} 16 \mathrm{em}$ 12h. No animal 15, a concentração permaneceu aumentada $\left(0,06 \mu \mathrm{g} \mathrm{L}^{-1}\right) \mathrm{em} 12 \mathrm{~h}$, mas retornou ao valor de repouso em $24 \mathrm{~h}$. Dos equinos que se observou aumento de cTnI, os animais 15 e 16 apresentaram, respectivamente, três e quatro contrações ventriculares prematuras (VPC) monomórficas e isoladas imediatamente após o exercício da $\mathrm{P} 2$. A cinética da cTnI sérica observada nesses animais foi semelhantemente encontrada por DURANDO et al. (2006), que relataram uma elevação discreta das concentrações de cTnI em 3 a 6 h, retornando aos níveis pré-exercício em 24 horas após exercício de alta intensidade e curta duração em esteira, em equinos de corrida de diversas raças.

Não foram utilizados animais que apresentaram alterações eletrocardiográficas de repouso que pudessem interferir no desempenho. Mesmo assim, pôde-se constatar a ocorrência de VPC isolados em três animais após o exercício, ocorrendo somente durante a recuperação do exercício, entre o final do exercício e 30min após o exercício. SENTA et al. (1970) acreditam que os VPC desse tipo, após atividade física, são funcionais e induzidos pelo reajuste do tônus nervoso autonômico e, portanto, benignos. Entretanto, em dois animais, foi possível a detecção do aumento de cTnI sérica, sugerindo a ocorrência de uma lesão miocárdica. Porém, pode-se considerar como uma lesão leve, uma vez que MICHIMA (2007) considera o limite normal de cTnI $0,3 \mathrm{~g} \mathrm{~L}^{-1}$, não indicando uma lesão cardíaca expressiva.

Segundo HOLBROOK et al. (2006), em equinos, não está definido um limite de concentração de cTnI que considere a gravidade da lesão cardíaca nem se os mecanismos ou doenças podem causar leve, moderada ou severa elevação desses níveis. Embora existam poucos relatos sobre a concentração de cTnI em equinos saudáveis, esses valores normais são variáveis. Essas diferenças são atribuídas aos diversos sistemas analisadores, que utilizam aminoácidos distintos para composição dos anticorpos monoclonais (DURANDO et al., 2006). Para MICHIMA (2007), a tentativa de extrapolar os resultados com aqueles apresentados na literatura, em equinos, é dificultada, uma vez que há divergência de metodologias e tipos de amostragem.

\section{CONCLUSÃO}

Os resultados do presente estudo permitem concluir que o exercício progressivo em esteira de alta velocidade promove estresse oxidativo, mas não é prevenido pela suplementação com 1.000UI de vitamina E nem por treinamento de 20 dias, além de não ocasionar lesão miocárdica expressiva. Entretanto, novas pesquisas devem ser realizadas a fim de esclarecer qual é a implicação desse estresse oxidativo sobre a saúde do animal e a cinética de liberação da troponina I cardíaca em decorrência do exercício. Além disso, essas pesquisas podem identificar os reais benefícios que a suplementação com vitamina $\mathrm{E}$ e o treinamento físico podem proporcionar ao animal.

\section{AGRADECIMENTOS}

Os autores agradecem à Fundação de Amparo à Pesquisa do Estado de São Paulo (FAPESP), pelo subsídio financeiro.

\section{COMITÊ DE ÉTICA E BIOSSEGURANÇA}

Projeto aprovado pela Câmara de Ética em Experimentação Animal (protocolo no 116/2005-CEEA) da Faculdade de Medicina Veterinária e Zootecnia, da Universidade Estadual Paulista, Campus de Botucatu.

\section{REFERÊNCIAS}

ARNAUD, J. et al. Simultaneous determination of retinol, $\alpha$ tocopherol and $\beta$-carotene in serum by isocratic high performance liquid chromatography. Journal of Chromatography, v.572, n.1-2, p.103-116, 1991.

ASCENSÃO, A. et al. Exercício e stress oxidativo cardíaco. Revista Portuguesa de Cardiologia, v.22, n.5, p.651-678, 2003.

ATALAY, M.; SEN, C.K. Physical exercise and antioxidant defenses in the heart. Annals of the New York Academy of Sciences, v.874, p.169-177, 1999.

AVELLINI, L. et al. Effect of exercise training, selenium and vitamin $\mathrm{E}$ on some free radical scavengers in horses (Equus caballus). Comparative Biochemistry and Physiology Part B, v.123, n.2, p.147-154, 1999

CHEN, Y. et al. Cardiac troponin T alterations in myocardium and serum in rats after stressful, prolonged intense exercise. Journal of Applied Physiology, v.88, n.5, p.1749-1755, 2000 .

DEATON, C.M. et al. Antioxidant supplementation and pulmonary function at rest and exercise. Equine Veterinary Journal, Supplement, v.34, p.58-65, 2002.

DE MOFFARTS, B. et al. Effect of oral antioxidant supplementation on blood antioxidant status in trained thoroughbred horses. Veterinary Journal, v.169, n.1, p.65-74, 
2005. Disponível em: <http://dx.doi.org/10.1016/j.tvjl.2003.12.012>. Acesso em: 21 set. 2005. doi:10.1016/j.tvjl.2003.12.012.

DURANDO, M.M. et al. Acute effects of short duration maximal exercise on cardiac troponin I in healthy horses. Equine and Comparative Exercise Physiology, v.3, n.4, p.217-223, 2006.

FREGIN, G.F. The equine electrocardiogram with standardized body and limb positions. Cornell Veterinarian, v.72, p.304324, 1982.

HOLBROOK, T.C. et al. Endurance exercise is associated with increased plasma cardiac troponin I in horses. Equine Veterinary Journal, v.36, supl, p.27-31, 2006.

KARATAS, F. et al. Determination of free malondialdehyde in human serum by high-performance liquid chromatography. Analytical Biochemistry, v.311, n.1, p.76-79, 2002.

KIRSCHVINK, N. et al. Investigation of blood oxidant/ antioxidant markers in healthy competition horses of different breeds. Equine Veterinary Journal, v.36, supl, p.239-244, 2006.

LEROUX, A.J. et al. Ventricular tachycardia associated with exhaustive exercise in a horse. Journal of the American Veterinary Medical Association, v.207, n.3, p.335-337, 1995.

MACHADO, L.P. et al. Susceptibilidade eritrocitária ao estresse osmótico em equinos da raça Árabe: efeito do exercício, treinamento e suplementação com vitamina E. Revista Universidade Rural Série Ciências da Vida, v.27, supl, p.134-136, 2007.

McMENIMAN, N.P.; HINTZ, H.F. Effect of vitamin E status on lipid peroxidation in exercised horses. Equine Veterinary Journal, v.24, n.6, p.482-484, 1992.

MICHIMA, L.E.S. Influência do exercício físico prolongado sobre a concentração sérica de troponina $I$ cardíaca e sobre a função cardíaca em cavalos de enduro. 2007. 94f. Tese (Doutorado em Clínica Veterinária) - Faculdade de Medicina Veterinária e Zootecnia, Universidade de São Paulo, SP.

NEUMAYR, G. et al. Effect of the "Race across the Alps" in elite cyclists on plasma cardiac troponins I and T. American Journal of Cardiology, v.89, n.4, p.484-486, 2002. Disponível em: $<$ http://dx.doi.org/10.1016/S0002-9149(01)02280-9>. Acesso em: 21 set. 2005. doi: 10.1016/S0002-9149(01)02280-9.
SENTA, T. et al. Effects of exercise on certain eletrocardiographic parameters and cardiac arrhythmias in the horse. A radiotelemetric study. Cornell Veterinarian, v.60, n.4, p.552-569, 1970.

SETIADI, D.H. et al. Vitamin E models. Can the anti-oxidant and pro-oxidant dichotomy of a-tocopherol be related to ionic ring closing and radical ring opening redox reactions? Journal of Molecular Structure, v.620, p.93-106, 2003.

VENDITTI, P. et al. Protection against ischemia-reperfusion induced oxidative stress by vitamin $\mathrm{E}$ treatment. Archives of Physiology and Biochemistry, v.107, n.1, p.27-34, 1999.

WHYTE, G.P. et al. Cardiac fatigue following prolonged endurance exercise of differing distances. Medicine and Science in Sports and Exercise, v.32, n.6, p.1067-1072, 2000.

WILLIAMS, C.A. Oxidative stress or oxidant/antioxidant disequilibrium in horses? A hot topic. Veterinary Journal, v.177, n.2, p.157-158, 2008. Disponível em: <http://dx.doi.org/ 10.1016/j.tvj1.2007.11.005>. Acesso em: 16 mar. 2010. doi: 10.1016/j.tvj1.2007.11.005.

WILLIAMS, C.A.; CARLUCCI, S.A. Oral vitamin E supplementation on oxidative stress, vitamin and antioxidant status in intensely exercised horses. Equine Veterinary Journal, v.36, supl, p.617-621, 2006.

WILLIAMS, C.A. et al. Antioxidant supplementation and subsequent oxidative stress of horses during an $80-\mathrm{km}$ endurance race. Journal of Animal Science, v.82, n.2, p.588-594, 2004.

WILLIAMS, C.A. et al. Apoptosis and antioxidant status are influenced by age and exercise training in horses. Journal of Animal Science, v.86, n.3, p.576-583, 2008. Disponível em: <http://dx.doi.org/10.2527/jas.2007-0585>. Acesso em: 16 mar. 2010. doi: $10.2527 /$ jas.2007-0585.

YEUM, K.J. et al. Biomarkers of antioxidant capacity in the hydrophilic and lipophilic compartments of human plasma. Archives of Biochemistry and Biophysics, v.430, p.97103, 2004.

YONEZAWA, L.A. et al. Marcadores cardíacos na medicina veterinária. Ciência Rural, v.40, n.1, p.222-230, 2010. Disponível em: <http://dx.doi.org/10.1590/S0103$84782009005000227>$. Acesso em: 16 nov. 2009. doi: 10.1590/S0103-84782009005000227. 